\title{
Etude économique de la production laitière dans la zone périurbaine de Bamako au Mali ${ }^{\star}$
}

\author{
S. Debrah ${ }^{1}$, K. Sissoko ${ }^{2}$, S. Soumaré ${ }^{3}$
}

DEBRAH (S.), SISSOKO (K.), SOUMARÉ (S.). Etude économique de la production laitière dans la zone périurbaine de Bamako au Mali. Revue Élev. Méd. vét. Pays trop., 1995, 48 (1) : 101-109

Le coût de production du lait frais dans trois systèmes de production laitière a été estimé à partir de données collectées sur 30 troupeaux aux alentours de Bamako au Mali, à des intervalles réguliers entre juillet 1989 et juin 1990. Dans le système de production intensive (concessions rurales), le coût de production du lait est estimé entre 110 et 195 F CFA// alors que dans le système extensif villageois et les parcs communaux, il est estimé respectivement entre 15 et $76 \mathrm{~F} \mathrm{CFA/l}$ et 62 et $408 \mathrm{~F} \mathrm{CFA/l}$. L'alimentation du bétail, le transport et les soins vétérinaires constituent les principaux éléments de dépenses. L'effectif optimal du troupeau, pour que les unités de production soient rentables, est de 8,9 et 25 vaches respectivement pour les parcs communaux, le système villageois et les concessions rurales. Le lait est vendu 100 à $250 \mathrm{~F}$ CFA/. Ainsi, avec ces niveaux de prix aux producteurs pratiqués avant la dévaluation du franc CFA, la production de lait est généralement rentable avec des bénéfices nets allant jusqu'à 106 F CFA/l. La récente dévaluation devrait encourager la production laitière locale afin de réduire le déséquilibre créé par la diminution des importations de produits laitiers.

Mots clés : Lait de vache - Production laitière - Système d'exploitation agricole - Coût de production - Economie de l'élevage - Commercialisation - Dévaluation - Enquête - Mali.

\section{INTRODUCTION}

L'ensemble des ressources animales des pays sahéliens était estimé à 19 milliards d'UBT ** en 1987 (1), soit approximativement 1,2 UBT par personne. Cependant, le niveau de consommation des produits de l'élevage, particulièrement celui du lait, reste bas. En Afrique de l'Ouest, la consommation moyenne de lait était de $13,3 \mathrm{~kg}$ ELL (Equivalent Lait Liquide) par habitant et par an (1). Dans une étude récente sur la consommation des produits laitiers conduite à Bamako au Mali, la consommation moyenne de lait pour l'ensemble des différents groupes de revenus était estimée à $12 \mathrm{~kg}$ ELL/hab. dont $60 \mathrm{p.} 100$ étaient constitués de produits laitiers importés (2). Ce niveau de consommation est faible, aussi bien par rapport aux normes internationales que par rapport à l'objec-

1. ICRISAT, West and Central Africa Regional Program, BP 320, Bamako, Mali.

2. Projet Production soudano-sahélienne, B.P 22, Niono, Mali.

3.Institut d'Economie rurale, B.P. 258, Bamako, Mali.

Reçu le 6.9.1994, accepté le 28.3.1995.

* Les recherches dans le cadre de cette étude ont été menées par le programme conjoint CIPEAIIER pendant que le premier auteur était employé au Centre international d'Elevage en Afrique (CIPEA), BP 60 , Bamako. tif du gouvernement malien d'atteindre $40 \mathrm{~kg}$ ELL/hab./an d'ici l'an $2000(4,5)$. Durant les 30 dernières années, la plupart des pays du Sahel ont compté sur les importations de produits laitiers pour satisfaire la demande croissante. Au Mali, ces importations ont augmenté de façon régulière à un taux annuel de 13,5 p. 100 entre 1961 et 1991, et leur valeur totale est passée de 436000 dollar's US en 1961 à 18,7 millions de dollars US en $1991(3,6)$.

Pour bien exploiter les ressources locales et réduire les importations de lait, il faut que la production soit rentable, ce qui motiverait les producteurs. Jusqu'ici, très peu d'études approfondies avaient été menées sur l'économie de la production du lait au Mali. L'objectif du présent travail est de corriger cette situation afin de permettre aux décideurs de prendre des mesures adéquates à partir d'informations fiables en matière de politique et de production des produits laitiers. Ceci favoriserait une augmentation de la production et de la consommation du lait et des produits laitiers au Mali. Cette étude constitue une partie d'une série d'études conjointes entreprises entre 1988 et 1991 par les chercheurs du CIPEA (Centre international pour L'Elevage en Afrique) et de l'IER (Institut d'Economie rurale) sur la production, la commercialisation et la consommation du lait et des produits laitiers au Mali. Bien qu'elle ait été réalisée avant la dévaluation du franc CFA survenue en janvier 1994, elle fournit des informations utiles qui pourraient conduire à la production efficiente de lait frais dans la zone périurbaine de Bamako afin de répondre aux demandes croissantes provenant de la production locale suite aux effets de la dévaluation.

\section{MATÉRIEL ET MÉTHODE}

L'étude a été conduite sur 30 unités de production laitière choisies parmi trois systèmes différents dans la zone de Bamako. Les données ont été collectées au niveau de chaque unité de production à un rythme hebdomadaire durant huit semaines au cours de chacune des trois saisons. La collecte des données de la saison sèche froide a eu lieu en novembre et décembre 1989, suivie de celle des données de la saison sèche chaude en mars et avril 1990, et enfin celle des données de la saison des pluies en juin et juillet 1990. La sélection de l'échantillon d'unités de production a été faite suivant la procédure multi-

\footnotetext{
** Unité Bétail Tropical : exemple de référence : bovin de $250 \mathrm{~kg}$
} 
étape. La première étape a consisté à choisir des zones à haut potentiel de production laitière, la deuxième à choisir les sites respectifs d'étude des trois systèmes de production. La dernière étape a consisté à sélectionner des unités laitières à partir des sites choisis.

\section{Description de la zone d'étude et des systèmes de production laitière}

La zone d'étude s'étend sur un rayon de $100 \mathrm{~km}$ autour de Bamako. Elle englobe le district de Bamako, une partie des cercles de Kati et de Koulikoro. Elle inclut donc la ceinture de pâturages identifiée comme zone recélant des potentialités pour la production du lait. Le climat est de type soudanien caractérisé par une saison pluvieuse de juin à octobre, une saison froide de décembre à février et une saison chaude de mars à mai. La zone est comprise entre les isohyètes 800 et $1100 \mathrm{~mm}$, avec une moyenne pluviométrique annuelle de $900 \mathrm{~mm}$, d'où l'appellation de "zone subhumide". Les principales caractéristiques des trois systèmes de production laitière décrits par Debrah et al. (2) sont résumées au tableau I.

Les unités de production villageoises sont localisées sur les terroirs agropastoraux des villages (à $25 \mathrm{~km}$ et plus de Bamako). Elles sont caractérisées par le système d'élevage extensif traditionnel basé essentiellement sur l'exploitation des races locales (la N'Dama et son produit de croisement avec le zébu Peul, la Méré) sur des pâturages naturels. Elles se caractérisent aussi par un très faible niveau de prélèvement laitier, de vente et de complémentation alimentaire qui n'existe d'ailleurs que chez quelques rares producteurs.

Les concessions rurales sont localisées aux abords immédiats de la ville de Bamako, dans un rayon dénommé "zone centrale" (allant jusqu'à $25 \mathrm{~km}$ en moyenne autour de Bamako). Ce sont des unités de production agropastorales appartenant surtout à des fonctionnairescommerçants résidant principalement dans le district de Bamako. La taille de ces exploitations est en général de 2 à 10 ha. Dans certaines concessions rurales, l'élevage coexiste avec d'autres spéculations telles que les cultures vivrières et les plantations d'arbres fruitiers. Dans d'autres, le système d'élevage est soit extensif traditionnel (identique à celui des unités de production villageoises), soit semi-intensif ou intensif (pratique de la complémentation). On note, dans la plupart des concessions rurales (environ 90 p. 100), une moyenne de 5 vaches de race exotique (principalement la Montbéliarde, la Rouge des steppes et la Méré exotique), avec un niveau relativement élevé de production et de vente de lait. Les producteurs des concessions rurales, du fait de leur situation socio-économique et de la proximité de la ville de Bamako, ont une plus grande facilité d'accès aux intrants et pour l'écoulement de leur production de lait.

Les parcs communaux se trouvent dans le district de Bamako (la ville est divisée en 6 communes). Pour chaque commune, il existe un ou plusieurs parcs dénommés parcs "communaux" qui sont, soit individuels, soit communs à plusieurs propriétaires. Les propriétaires de ces parcs, à la différence de ceux des concessions rurales, ne disposent pas d'espace à droit d'usage individuel et le nombre d'animaux est beaucoup plus faible. Le système d'élevage de type semi-intensif est basé sur l'exploitation des pâturages naturels et des races locales, avec un niveau et une qualité de complémentation plus faibles que ceux des concessions rurales.

\section{Choix des sites d'enquête et des unités laitières-échantillons}

Les villages situés sur les axes retenus ont fait l'objet d'une description socio-économique. Sur cette base, et en fonction de la distance qui sépare les villages des axes principaux, 9 villages (Sanankoroba, Dialakoroba, Falan, Falanikoungo, Kandian, Ouéléssébougou, Tinguélé, Siby et Bancoumana) ont été présélectionnés. D'autres critères de choix ont été ensuite utilisés pour la sélection finale : accessibilité, facilité de communications avec Bamako, existence ou proximité de poste vétérinaire et de marchés, existence de sources d'abreuvement, accessibilité aux pâturages, proximité des parcs du village, possibilité de logement pour l'enquêteur, existence de projets ou autres services dans la zone. Sur la base de ces critères, 3 villages sur les 9 ont été retenus.

Le choix des parcs communaux dans le district de Bamako a été précédé d'une visite à la direction du District vétérinaire et dans huit parcs situés respectivement à N'Tomikorobougou, Lafiabougou, Sébénikoro, Missabougou, Boulkassoumbougou, Faladié, N'Komi et Bako-Djikoroni. Le but de ces visites était d'obtenir des informations sur ces parcs, aussi bien sur le plan de la gestion que sur celui des problèmes zootechniques majeurs. Sur cette base, deux localités (Missabougou et Sébénikoro) ont été retenues. Les critères' du choix étaient les types de parcs (commun ou individuel), l'importance du cheptel bovin, l'accessibilité et la situation géographique du parc.

L'étude de diagnostic sur les concessions rurales (2) a servi de base pour le choix des sites à étudier. Ainsi, trois Iocalités (Tienfala, Missabougou et Sanankoroba) sur les 15 sites identifiés ont été retenues. Les principaux critères étaient: : la situation, la répartition géographique et l'importance du nombre de concessions rurales regroupées par localité. Le choix de Tienfala a été motivé par l'importance de ce regroupement dans cette localité située sur l'axe Bamako/Koulikoro. Quant aux localités de Sanankoroba et de Mișsabougou, leur choix a été dicté respectivement par la présence de concessions rurales à proximité du système villageois et des parcs communaux. Cela permet d'avoir des éléments de comparaison entre différents systèmes d'élevage se côtoyant sur le même terroir agropastoral. 
TABLEAU I

Caractéristiques des principaux systèmes de production laitière en zone périurbaine de Bamako
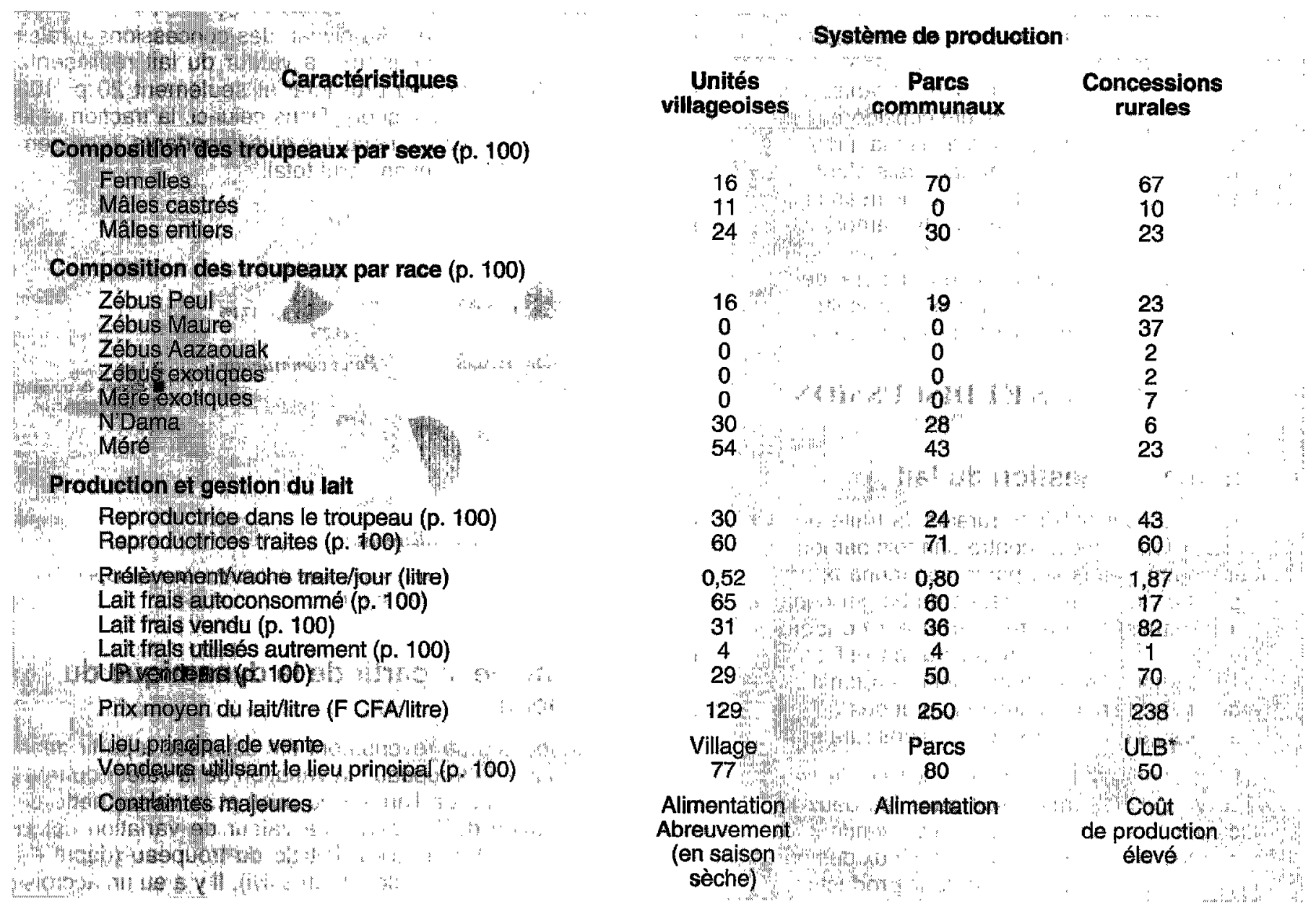

Source : Debrah, Sissoko, Soumaré, Traoré, Diagnostic de la production laitière aux environs de Bamako. 1989.

En ce qui concerne le choix des unités laitières-échantillons, il s'agissait de choisir certaines unités de production parmi les unités villageoises, parcs communaux et concessions rurales localisés au niveau des sites d'enquêtes retenus. Le principe appliqué a été la participation à l'enquête sur la base du volontariat. Les objectifs de l'étude et la méthodologie de travail de l'équipe (manipulations des animaux telles que le marquage, la pesée, le contrôle laitier, l'exploration rectale, la prise de sang, etc.) ont été expliqués aux éleveurs des différents sites d'enquête. Après une telle sensibilisation, des listes de volontaires ont été dressées et trois principaux critères ont été utilisés pour le choix de l'échantillon des unités de production laitière : le nombre de génotypes (races) représentés dans le troupeau (la préférence a été donnée aux troupeaux contenant plus d'un génotype) ; le nombre de reproductrices dans le troupeau qui doit être supérieur ou égal à 3 ; la taille du troupeau en relation avec les effectifs de bovins dans la localité (grand, moyen ou petit troupeau). Au total, 13 unités de production villageoises, 6 parcs communaux (comprenant 5 troupeaux individuels et un troupeau commun appartenant à 10 personnes) et 11 concessions rurales ont été retenus.

\section{Collecte des données et méthodologie d'analyse}

Les 30 unités laitières-échantillon's (avec un total de 2000 bovins environ) réparties dans les trois systèmes. de production ont été suivies à un rythme hebdomadaire durant la période de juillet 1989 à juin 1990 par six observateurs. Ces derniers résidaient chacun au niveau d'un site de la zone d'étude et étaient sous la supervision. d'une équipe pluridisciplinaire. Les questionnaires ont été intégrés de manière que les observateurs puissent se charger de la collecte de l'ensemble des informations' sectorielles (socio-économie, zootechnie, reproduction, écologie).

Dans l'analyse, le bénéfice net a été calculé en déduisant le total des coûts variables de production du revenu total 
obtenu pour chaque unité de production. L'estimation du revenu global d'activité de l'élevage bovin est basée essentiellement sur la valeur du lait, les éléments de la dynamique des troupeaux, la traction et la production de fumier. Pour l'estimation des coûts variables de production, les éléments suivants ont été considérés : frais vétérinaires, achat d'aliments, coûts de la main-d'oeuvre, frais de transport des intrants et du lait, frais d'entretien et de réparation des équipements et des diverses installations. Dans cette estimation, la valeur de l'amortissement de l'ensemble des investissements, tels que bâtiments et équipements réalisés dans le cadre général de l'élevage, n'a pas été considérée à cause des difficultés d'évaluation dues au manque de fiabilité des données collectées.

\section{RÉSULTATS ET DISCUSSION}

\section{Production et gestion du lait}

Au niveau des concessions rurales, la traite des laitières se fait deux fois par jour, contre une fois par jour dans les villages comme dans les parcs communaux. Une partie de la production totale est cédée au berger comme paiement en nature, une autre partie est consommée au niveau de la ferme et le reste du lait est destiné à la vente. Les quantités vendues varient en fonction du système de production, mais les plus grandes quantités sont enregistrées au niveau des concessions rurales.

Le lait est vendu soit à Bamako, soit sur le lieu de production (village ou ferme), soit dans les deux lieux. Les producteurs des concessions rurales vendent 90 p. 100 de leur production totale à Bamako. Ceux des parcs communaux vendent 57 p. 100 de leur production totale à Bamako et 38 p. 100 sur le lieu de production, tandis que ceux du système villageois vendent 80 p. 100 de leur production dans le village. Les principales catégories d'acheteurs sont les consommateurs directs, les intermédiaires et une usine de traitement de lait paraétatique, l'Union laitière de Bamako (ULB). Les producteurs des concessions rurales vendent 90 p. 100 de leur production à l'ULB, tandis que les parcs communaux vendent $76 \mathrm{p}$. 100 de leur production aux intermédiaires et que $96 \mathrm{p}$. 100 de la production des villages sont vendus directement aux consommateurs.

\section{Estimation du revenu global par système de production}

La figure 1 montre, par système de production, les éléments qui composent les bénéfices bruts, c'est-à-dire la valeur du lait, celle de l'exploitation du troupeau, et celle du fumier et de la traction. La valeur du lait a été estimée en multipliant le prix moyen pratiqué par la quantité totale de lait produit (y compris la part consommée par les veaux). La valeur moyenne pour les trois systèmes confondus se situe à $10771 \mathrm{~F}$ CFA/unité de production par jour. Les valeurs moyennes respectives réalisées par les concessions rurales, les parcs communaux et les unités villageoises sont de 23276,5314 et 2708 F CFA/unité laitière/j. Au niveau des concessions rurales et des parcs communaux, la valeur du lait représente 58 p. 100 du revenu brut total et seulement 20 p. 100 dans le système villageois. Dans celui-ci, la traction et le fumier étaient les éléments les plus importants, représentant 50 p. 100 du revenu brut total.

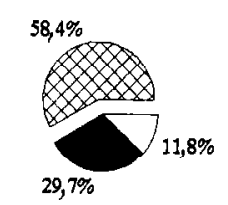

Concessions rurales

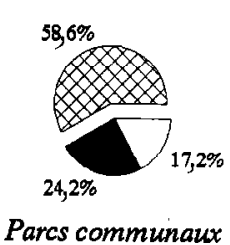

Parcs communaux $\square$ Lait $\square$ Fumier et traction
Exploit. du troupeau

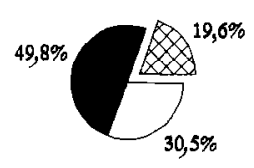

Unités villageoises

Figure 1.: Importance des composantes du bénéfice brut des exploitations laitières par système de production.

\section{Revenu net à partir de la dynamique du troupeau}

Deux sources de revenus ont été estimées à partir de la dynamique du troupeau : la variation de la valeur du troupeau au cours de l'année du suivi et la valeur nette de l'exploitation du troupeau. La valeur de variation est la différence entre la valeur initiale du troupeau (début du suivi) et sa valeur finale (fin du suivi). II y a eu un accroissement net de l'effectif du troupeau de 46,7 et 28 animaux respectivement au niveau des concessions rurales, des parcs communaux et des unités villageoises au cours de l'année. Les valeurs de cet accroissement de l'effectif sont estimées à 8319,1414 et 5042 F CFA respectivement pour les concessions rurales, les parcs communaux et le système villageois.

La valeur nette de l'exploitation du troupeau a été estimée en prenant en compte les ventes des animaux sur pied et les abattages moins la valeur des animaux volés ou égarés. Cela représente une valeur nette de 11854 F CFA au niveau des concessions rurales représentant 29,7 p. 100 du bénéfice brut. Pour les parcs communaux et le système villageois, la valeur nette se situe respectivement à $2198 \mathrm{~F} \mathrm{CFA}$ et $6872 \mathrm{~F} \mathrm{CFA}$ $(49,8$ p. 100) du bénéfice brut des exploitations laitières.

\section{Valeur du fumier et de la traction animale}

Pour estimer la valeur du fumier déposé pendant la durée du suivi, une version de la formule proposée par l'Institut sénégalais de Recherche agricole (ISRA) a été utilisée. La quantité de fumier déposée est calculée à partir de la 
taille du troupeau exprimée en UBT (Unité Bétail Tropical). En supposant que 50 p. 100 de la quantité du fumier déposé sont utilisés comme engrais, à raison de $10000 \mathrm{~F}$ CFA la tonne, la valeur du fumier a été estimée respectivement à 469, 1545 et 3435 F CFA par jour et par unité de production dans les concessions rurales, les parcs communaux et le système villageois pendant l'année de suivi.

La valeur de la traction a été estimée en multipliant le nombre de jours de travail des boeufs de labour par un prix unitaire de 2500 F CFA par jour qui représente le coût moyen de la location d'une unité de traction. Ainsi, les valeurs du travail sont estimées à 26,10 et 779 F CFA/j et par unité de production respectivement dans le système villageois, les concessions rurales et les parcs communaux. Au niveau des concessions rurales, le fumier et la traction contribuent pour 11,8 p. 100 dans le revenu brut tandis que leur contribution est de 17,2 et $30,5 \mathrm{p}$. 100 respectivement au niveau des parcs communaux et des unités de production villageoises.

\section{Estimation du coût de fonctionnement par système}

La figure 2 résume l'importance des différents postes de dépenses (aliments, transports, main-d'oeuvre, médicaments et coûts divers) dans les coûts de fonctionnement par système de production. La rubrique coûts divers concerne les frais de réparation, de réfection et d'entretien des bâtiments et équipements ainsi que l'achat de petits matériels tels que les abreuvoirs, les mangeoires, les cordes et les bidons. Au niveau des concessions rurales et des parcs communaux, ce sont les coûts d'alimentation qui représentent la plus grande part du budget de fonctionnement, estimés à 50,7 p. 100. Les dépenses d'alimentation au niveau des concessions rurales concernent l'achat d'aliment du bétail (tourteaux de coton et d'arachide) auprès d'unités agro-industrielles locales (HUICOMA et Grands Moulins du Mali). Les propriétaires de concessions rurales achètent aussi d'autres aliments comme la farine basse de riz et de blé. Les parcs communaux et les unités villageoises dépendent, en majeure partie, des résidus de récolte et de fourrages (fanes de niébé et d'arachide), de la paille de brousse et, occasionnellement, le sel et la mélasse sont utilisés comme suppléments alimentaires. Les coûts de transport constituent l'élément le plus élevé, après l'alimentation, pour les concessions rurales (28,7 p. 100). Ils comprennent le coût de transport de l'aliment et d'autres intrants aussi bien que le coût de transport du lait pour la vente à Bamako. Les parcs communaux et les unités villageoises dépensent moins en frais de transport mais beaucoup en main-d'oeuvre, laquelle est du reste essentiellement constituée par le clan familial.
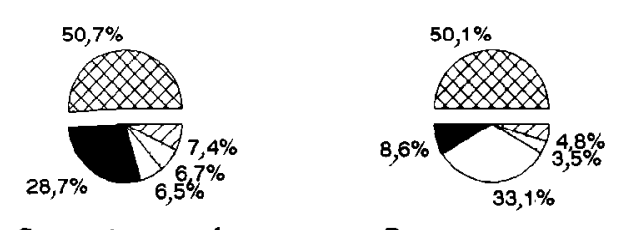

Concessions rurales

Parcs communaux

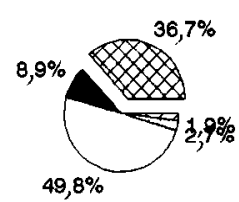

囚 Aliments

Transports

Main-d'oeuvre

$\square$ Divers

Unités villageoises

Figure 2 : Importance des postes de dépenses dans le cô̂t de fonctionnement total par système de production.

\section{Rentabilité économique de la production laitière par système}

\section{Estimation du coût unitaire de la production laitière}

Dans une unité d'élevage, les coûts de production du lait font partie intégrante du coût de fonctionnement global de toutes les spéculations entreprises par l'éleveur. Ainsi, la part des coûts de production du lait dans le coût total de fonctionnement a été estimée sur la base de la valeur de la production du lait dans le produit brut de l'élevage. Le coût unitaire de production laitière (F CFA/litre) correspond donc au rapport du coût associé directement à la production du lait sur la quantité totale de lait produit. La performance économique d'une unité de production est le reflet de la qualité de sa gestion. Elle est jugée à travers trois paramètres : la marge bénéficiaire, le coût unitaire de production et la productivité. Une exploitation laitière bien gérée doit produire du lait à moindre coût avec un niveau de production laitière et une marge bénéficiaire satisfaisants. Ainsi, pour chacun des trois systèmes de production laitière, ces paramètres ont été analysés et comparés (tabl. II). Si le lait est vendu entre 100 et 250 F CFAll, et le coût de production estimé à un montant entre 44 et $153 \mathrm{~F} \mathrm{CFA/l,} \mathrm{la} \mathrm{marge} \mathrm{nette} \mathrm{varie} \mathrm{d'une} \mathrm{perte}$ de $3 \mathrm{~F} \mathrm{CFA/l} \mathrm{pour} \mathrm{les} \mathrm{concessions} \mathrm{rurales} \mathrm{à} \mathrm{un} \mathrm{profit} \mathrm{de}$ 106 F CFA/l dans les unités villageoises. Pour un prix de vente du lait compris entre 150 et 250 F CFA/l, 45 p. 100 des unités de production ( 5 unités de production sur 11) au niveau des concessions rurales et 16 p. 100 (1 producteur sur 6) au niveau des parcs communaux produisent le lait à perte. Aucun producteur d'unités villageoises ne produit le lait à perte.

Bien que dans l'ensemble la production de lait semble rentable au niveau de tous les systèmes, il y a une grande disparité entre les unités de production prises de façon individuelle. Dans le tableau III, l'unité laitière la mieux gérée est comparée à la moins bien gérée dans chacun des systèmes, en vue de montrer l'impact de la gestion sur la productivité, la production, le coût et le profit par litre. Le niveau de production de lait dans la 
S. Debrah K. Sissoko S. Soumaré

TABLeau II

Rentabilité économique de la production laitière dans trois systèmes de production laitiers autour de Bamako

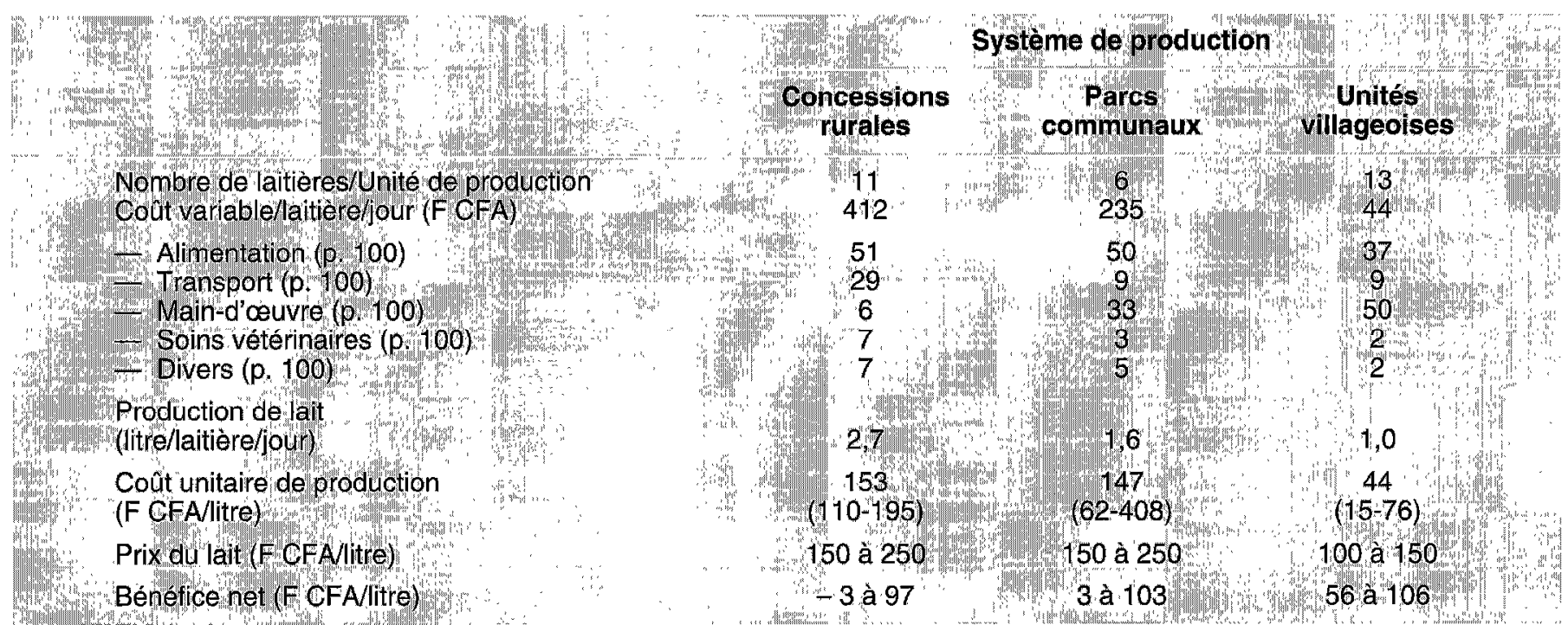

N.B. Les chiffres entre parenthèses représentent les limites de la variation du coût unitalre de productlon. Source : Données des enquêtes sur 30 troupeaux autour de Bamako de juillet 1989 à juin 1990 .

Tableau III

Profil selon la qualité de la gestion dans les trois systèmes laitiers autour du Bamako

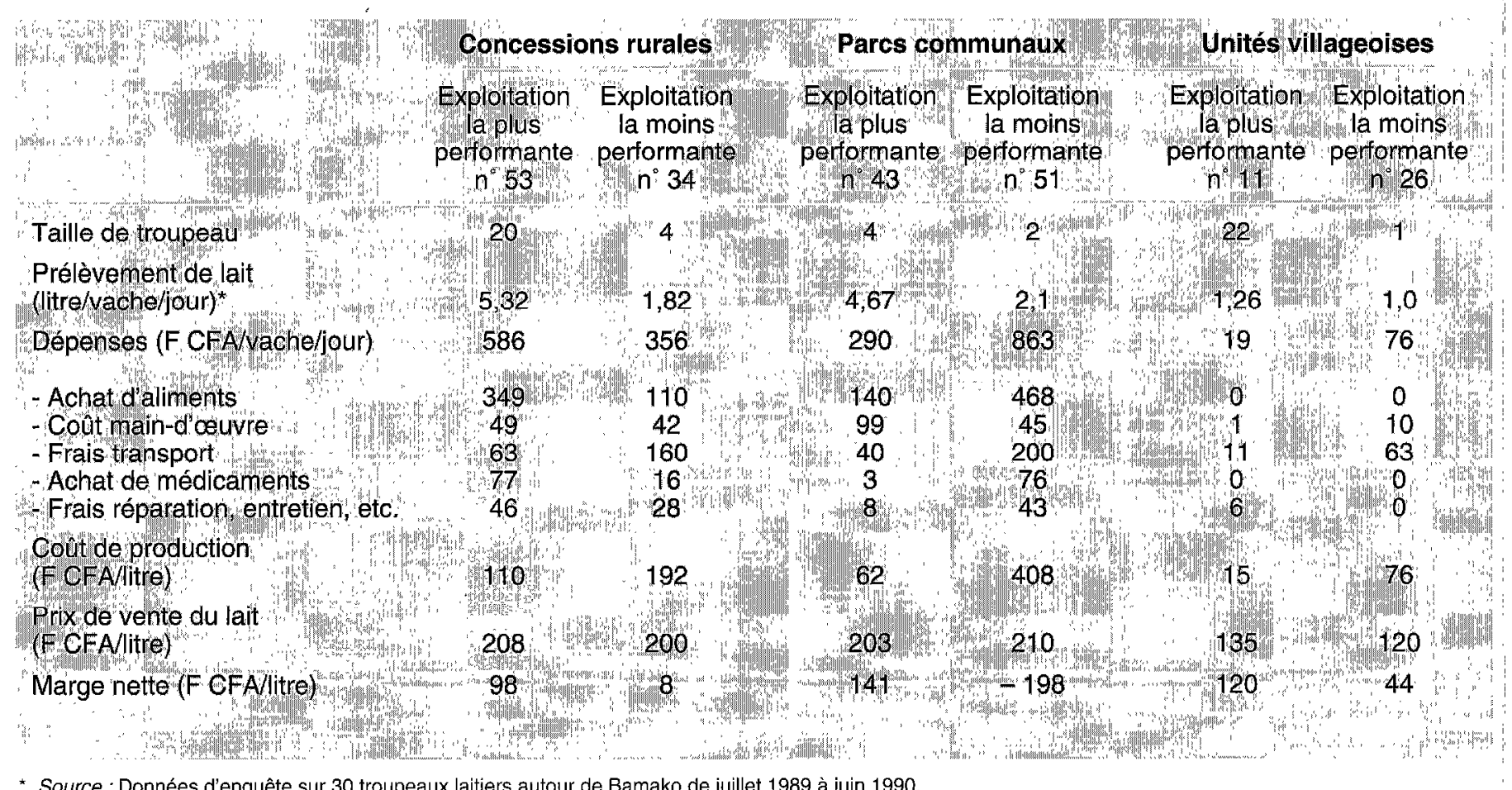


meilleure unité laitière parmi les concessions rurales (troupeau $n^{\circ} 53$ ) se situe à $5,33 \mathrm{l} /$ vache/j. Cette unité se caractérise aussi par le coût unitaire de production le plus bas (110 F CFA/l) comparativement à la moins performante (troupeau $n^{\circ} 34$ ) où le prélèvement de lait se situe à $1,82 \mathrm{l} /$ vache/j avec un coût unitaire de $195 \mathrm{~F} \mathrm{CFA/l}$. Au niveau des parcs communaux, l'unité la plus performante (troupeau $n^{\circ} 43$ ) a produit en moyenne 4,67 l/vache/j à un coût moyen de $62 \mathrm{~F} \mathrm{CFA/l,} \mathrm{comparativement} \mathrm{au} \mathrm{parc}$ communal le moins performant (troupeau $n^{\circ} 51$ ) où le prélèvement est de $2,1 \mathrm{l} / \mathrm{vache} / \mathrm{j}$ avec un coût unitaire de 408 F CFA/l. Pour les unités laitières villageoises, la même tendance a été aussi constatée en matière de productivité et de coût de production entre l'unité villageoise la plus performante (troupeau $n^{\circ} 11$ ) et l'unité la moins performante (troupeau $n^{\circ} 26$ ).

\section{Perspectives d'amélioration de l'efficacité dans la production}

L'alimentation, la main-d'œuvre, le transport et les soins vétérinaires sont les éléments les plus importants des coûts de production indiqués dans la figure 2 . Afin d'obtenir une production plus efficace de lait, ces facteurs doivent être utilisés de manière qu'il en résulte une production optimale par vache. Dans la meilleure unité de production des concessions rurales, les dépenses complémentaires sur l'alimentation représentent 60 p. 100 du total des coûts, la main-d'oeuvre 8 p. 100, les transports 11 p. 100 et les soins vétérinaires 13 p. 100 . Cette exploitation obtient une production journalière de 5,32 I de lait par vache.

En revanche, l'unité de production la moins performante a dépensé seulement 4 p. 100 pour les soins vétérinaires 12 p. 100 pour la main-d'oeuvre, 30 p. 100 pour l'alimentation, mais 45 p. 100 pour le transport, et a obtenu une production journalière de 1,82 I par vache. Ainsi, on n'insistera jamais assez sur l'importance de l'alimentation et de la bonne santé pour le maintien de la productivité. Les autres coûts liés à la production du lait, tels que la main-d'oeuvre, le transport et les opérations diverses dépendent de la taille du troupeau.

Certaines exploitations élèvent de nombreux animaux rendant ainsi les opérations de production et la gestion inefficaces, pendant que d'autres possèdent de si petites tailles de troupeaux que les frais d'exploitation par tête sont élevés. Le tableau IV récapitule l'information sur la relation entre la taille du troupeau et le coût unitaire de production pour les 30 unités de production de l'échantillon. Dans les concessions rurales, les exploitations ayant entre 3 et 14 vaches laitières produisent environ 344 à $611 \mathrm{l} /$ mois et atteignent des coûts de production de 175 à 195 F CFAll. Celles ayant entre 11 et 35 vaches laitières produisent à moins de $152 \mathrm{~F} \mathrm{CFA} / \mathrm{l}$.

Les figures 3,4 et 5 montrent les coûts moyens de production de lait par vache selon la taille du troupeau respectivement dans les concessions rurales, les parcs communaux et le système villageois de production. Au niveau des concessions rurales, le coût minimal de production est de
$110 \mathrm{~F} \mathrm{CFA/l} \mathrm{fourni} \mathrm{par} \mathrm{le} \mathrm{troupeau} n^{\circ} 53$. II correspond à une production de $1740 / / /$ mois pour un effectif de 25 vaches. Soixante-quatre pour cent des producteurs de concessions rurales maintiennent actuellement un troupeau de petite taille pour avoir l'économie d'échelle. Au contraire, 27 p. 100 maintiennent de trop grandes tailles pour produire de façon efficace. Au niveau des parcs communaux et des systèmes villageois, la plupart des producteurs se situent dans la zone de la courbe où le coût de production est élevé à cause de la' trop grande taille des troupeaux. En se basant sur l'échantillon, la taille idéale de troupeau dans les concessions rurales se situe entre 21 et 29 vaches, ce qui donne un coût unitaire de production entre 110 et $130 \mathrm{~F} \mathrm{CFA} /$. De la même manière, le nombre idéal de vaches laitières dans les parcs communaux se situe entre 6 et 10 vaches tandis qu'au niveau du système de production villageois, il se situe entre 6 et 12 .

\section{Dévaluation du franc CFA et perspectives de production du lait}

Le franc CFA a été dévalué à 50 p. 100 de sa valeur initiale dans les pays francophones le 12 janvier 1994. Lés effets d'une telle dévaluation découragent du point de vue théorique les importations tandis que la production interne, de même que les exportations, sont favorisées. Dans le secteur du lait, le recours aux produits laitiers importés pour faire face à la demande ne sera plus réalisable, ce qui fait qu'il devient impérieux d'accroître la production interne. Au cours de la période précédant la dévaluation, les frais de l'aliment du bétail, du transport et des soins vétérinaires constituaient les principaux éléments du coût de production. On s'attend à ce que le prix de l'aliment du bétail, qui dépend en grande partie de la disponibilité en sous-produits du coton et de l'arachide, baisse par suite de l'augmentation de l'offre. Les frais de transport et de soins vétérinaires, tributaires en grande partie des importations, devraient augmenter. Cependant, la demande de lait et de produits laitiers locaux doit s'intensifier par suite de l'augmentation du prix des produits laitiers importés et de la baisse des importations. Cela se traduira par des prix aux producteurs plus élevés qu'avant la dévaluation, créant ainsi plus d'incitation pour ces derniers.

\section{CONCLUSION}

Les résultats de l'étude montrent que la production de lait est rentable pour l'ensemble des trois systèmes de production étudiés. Cependant, le niveau de production de lait, le coût de production et le profit par litre de lait varient de façon substantielle en fonction du système étudié. Les résultats obtenus font aussi ressortir des différences dans la performance économique des systèmes de production de l'échantillon étudié. Ceci dépend principalement de l'efficacité des producteurs dans la gestion des activités au niveau de leur unité respective. Ainsi, il existe une taille idéale de troupeau pour chaque système qui est de 21 à 29 vaches dans les concessions rurales, 6 à 10 dans les parcs communaux et 6 à 12 en système villageois. 
S. Debrah K. Sissoko S. Soumaré

Tableau IV

Relation entre la taille du troupeau laitier, la production du lait et le coût unitaire de production

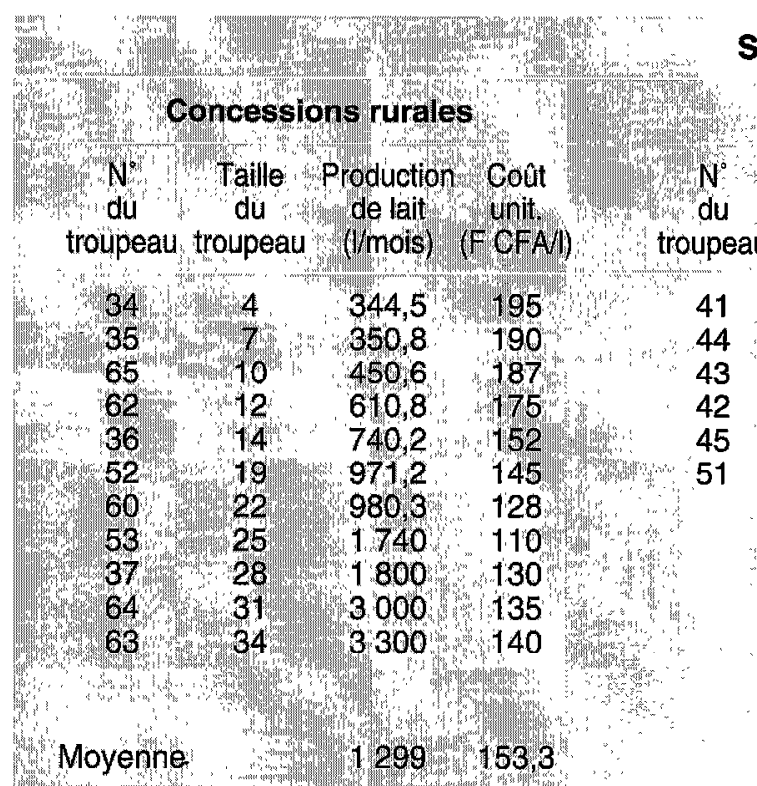

Système de production

Parcs communaux

dü de lait unit: roupeau (I/mois) (F CFAl)
Taille Production colt

Unites Villageoises

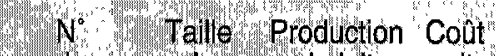
troupeau troupeau (I/mois) (FOFAI)

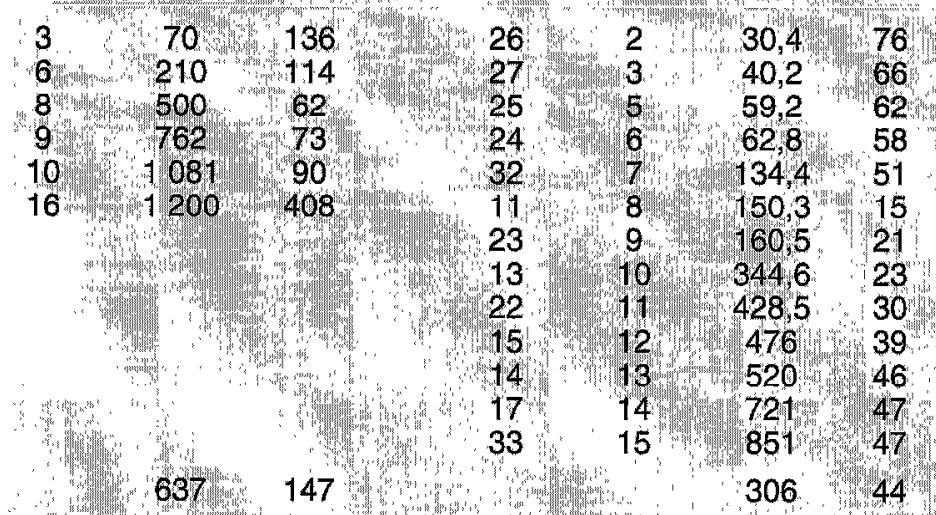

Source : Données d'enquêtes sur 30 troupeaux laitiers autour de Bamako, de juillet 1989 à juin 1990.

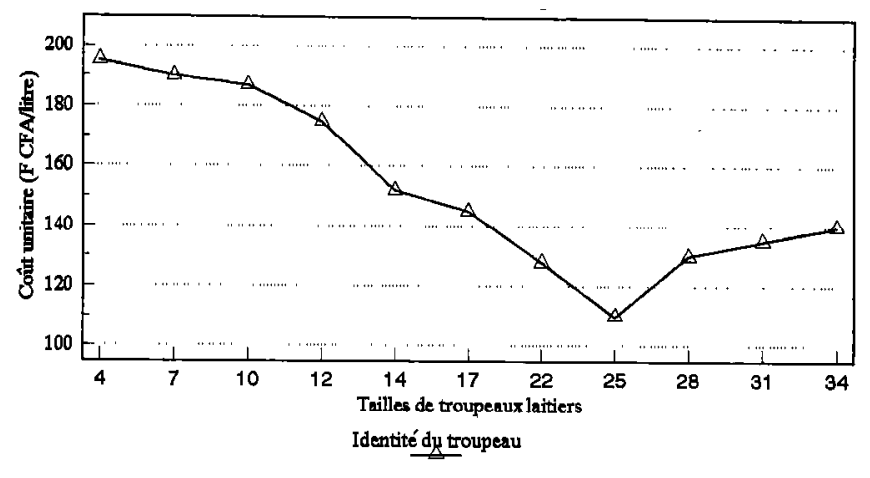

Figure 3 : Relation entre la taille du troupeau laitier et le coult unitaire de production de lait dans les concessions rurales. (Source : Données d'enquête sur 11 troupeaux laitiers de juillet 1989 à juin 1990).

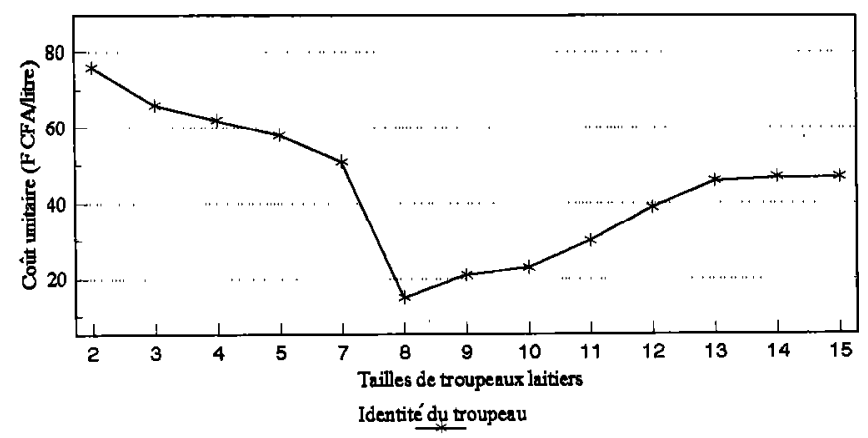

Figure 5 : Relation entre la taille du troupeau laitier et le coût unitaire de production de lait dans les unités villageoises. (Source : Données d'enquête sur 13 troupeaux laitiers de juillet 1989 à juin 1990).

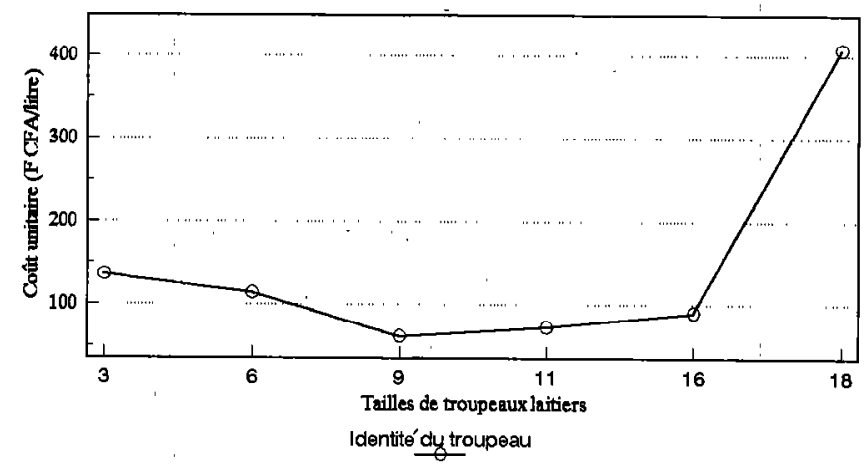

Figure 4 : Relation entre la taille du troupeau laitier et le coût unitaire de production de lait dans les parcs communaux. (Source : Données d'enquête sur 6 troupeaux laitiers de juillet 1989 à juin 1990).

\section{REMERCIEMENTS}

Les auteurs remercient M. D. Sanogo pour sa collaboration lors de la révision du document et tous ceux qui, dans l'anonymat, ont contribué à la publication de cet article.

\section{BIBLIOGRAPHIE}

1. CIPEA (Centre International pour l'élevage en Afrique), 1994. Recueil statistique sur la production animale en Afrique. Addis Abeba, Ethiopie, CIPEA, $63 \mathrm{p}$.

2. DEBRAH S., SISSOKO K., SOUMARÉ S., TRAORÉ M., 1989. Diagnostic de la production laitière aux environs de Banlako. In: Session 1989 de la commission technique de l'IER, Bamako, Mali, 18 p. 
3. F $\Lambda \mathrm{O}$ (Food and $\Lambda$ gricultural Organization of the United Nations), 1994. Agrostat-PC Database, Agrostat PC, 1993, Rome, Italy, FAO.

4. MEE (Ministère de l'Environnement et de l'Elevage), 1989. Commercialisation-distribution des produits laitiers et financement des actions. In Séminaire sur la filière lait au Mali, 13 au 16 juin 1989, Bamako, Mali, 21 p.

DEBRAH (S.), SISSOKO (K.), SOUMARE (S.). Economic study of dairy production in the area around Bamako (Mali). Revue Élev. Méd. vét. Pays trop., 1995, 48 (1): 101-109

The cost of fresh milk production in three different dairy production systems was estimated from data collected from 30 herds in the Bamako area (Mali) at regular intervals between July 1989 and June 1990. In the intensive dairy production system ("concessions rurales") milk production is estimated to cost between 110 and 195 F CFA/l while in the extensive village system ("système villageois") and the "parcs communaux", the cost of production is estimated respectively between 15 and 76 F CFA/l and 62 and 408 F CFA/l. Feeding, transportation and veterinary care constituted the major cost components. The optimum dairy herd sizes at which milk production is profitable are estimated at 8,9 and 25 respectively in the communal herds, the extensive village system and the intensive, peri-urban systems. At the producer price levels of between 100 and 250 F CFA before the devaluation of the CFA franc, milk production was generally considered a profitable entreprise with net profit of up to 106 F CFA/l possible. It is considered that the recent devaluation of the CFA franc will encourage domestic dairy production to respond to the additional demand created by reduced dairy imports.

Key words: Cow milk - Milk production - Farming systems - Production cost - Livestock economics - Marketing - Devaluation - Survey - Mali.
5. MRNE (Ministère chargé des Ressources naturelles et de l'Elevage), 1985. Politique Laitière. Bamako, Mali, MRNE.

6. VON MASSOW V.H. 1986. Importation de produits laitiers et politique d'importation au Mali. Effets sur le secteur laitier dans la région de Bamako. Addis Abeba, Ethiopie, CIPEA, 77 p. (Document de travail LPU $\left.n^{\circ} 8\right)$

DEBRAH (S.), SISSOKO (K.), SOUMARE (S.). Estudio económico de la producción de leche en la zona peri-urbana de Bamako en Mali. Revue Élev. Méd. vét. Pays trop., 1995, 48 (1): 101-109

El costo de la producción de leche fresca en tres sistemas de producción lechera fue estimado a partir de datos recolectados en $\mathbf{3 0}$ hatos alrededor de Bamako en Mali, en intervalos regulares entre julio 1989 y junio 1990. En el sistema de producción intensiva de leche (concesiones rurales), el costo de producción es estimado entre 110 y 195 F CFA por litro, siendo que en el sistema extensiva aldeaño y en los grupos comunales, este es estimado respectivamente entre 15 y 76 F CFA por litro y entre 62 y 408 F CFA por litro. La alimentación del ganado, el transporte y los servicios veterinarios constituyen los principales elementos de los gastos. El efectivo optimo del hato para que las unidades de producción lechera sean rentables es de 8,9 y 25 vacas respectivamente para los grupos comunales, el sistema aldeaño y las concesioncs rurales. La leche es vendida 100 a 250 F CFA por litro. De esta forma, con estos niveles de precios praticados por los productores antes de la devaluación del F CFA, la producción de leche es generalmente rentable con beneficios netos que alcanzan 106 F CFA por litro. La reciente devaluación deberia fomentar la producción lechera local con el fin de satisfacer el desequilibrio creado por la reducción de las importaciones de productos lacteos.

Palabras clave : Leche de vaca - Producción lechera - Sistema de exploitación - Costo de producción - Economía de la cría - Mercadeo Devaluación - Encuesta - Mali. 